\title{
Manuscritos caligráficos en fondos antiguos: análisis del ms. Göttingen Schönschriften (ca. 1560)
}

\author{
Diego Navarro*
}

Artículo recibido:

17 de agosto de 2016

Artículo aceptado:

9 de marzo de 2017

\section{Resumen}

Se analizan las principales características del manuscrito caligráfico titulado Schönschriften (=Bellas escrituras) hallado en la biblioteca histórica de la Universidad de Göttingen (Alemania). Se aplica un método basado en los principios de la descripción material para desentrañar aspectos formales, virtuosismo técnico y estético, y circunstancias históricas de producción y uso de las "Artes de escribir” de los siglos XVI y XVII. Estos ejemplos muestran la necesaria integración entre investigación y práctica caligráfica. Sus resultados se pueden aplicar a la mejora de la formación bibliotecológica y paleográfica de personal responsable de la gestión de fondo antiguo.

\footnotetext{
Departamento de Biblioteconomía y Documentación,

Universidad Carlos III de Madrid, España.

dnavarro@bib.uc3m.es

INVESTIGACIÓN BIBLIOTECOLÓGICA, vol. 32, núm. 76, julio/septiembre, 2018, México, ISSN: 2448-8321 pp. 149-165
} 
Palabras clave: Caligrafía; Descripción Material; Paleografía; Colecciones Especiales.

Calligraphic manuscripts in special collections: analysis of ms. Göttingen Schönschriften (ca.1560)

Diego Navarro

\begin{abstract}
Main characteristics of the calligraphic manuscript entitled Schönschriften (beautiful writings) discovered in the Historical Library, University of Göttingen (Germany) are analyzed here. A method based on the principles of material description is developed here to find out details of its formal structure, technical and aesthetical calligraphic mastery and historical circumstances of production and use. This example is part of a large tradition of handwriting and penmanship existing during the 16-17th centuries and displays the convenient integration between academic research and calligraphic practice to achieve improvements in the specialized training on paleography skills of staff responsible for special collections.
\end{abstract}

Keywords: Calligraphy; Material Description, Paleography, Special Collections.

\title{
INTRODUCCIÓN
}

$\mathrm{E}^{\mathrm{n}}$ el transcurso de las investigaciones sobre las múltiples dimensiones y niveles que definen la cultura de lo escrito, la búsqueda de testimonios originales que acrediten la excelencia del trabajo caligráfico en Europa desde los siglos XV en adelante ha deparado algunas sorpresas de interés. Una de las más recientes se sitúa en la Biblioteca Histórica de la Universidad de Göttingen (Baja Sajonia, Alemania). Entre sus fondos manuscritos se localizó un códice inédito, fiel exponente de las llamadas genéricamente "artes de escribir", que se dieron con fuerza a partir del siglo XV. Éstas definen prácticas escriturarias, formativas y normalizadoras caligráficas de altísimo nivel y competencia, prácticas rastreables, por otra parte, sin mucha dificultad 
por toda la geografía archivística y bibliotecaria internacional. ${ }^{1}$ A pesar del amplio listado de maestros calígrafos que jalonan la historia del arte de la escritura desde el siglo XVI en toda Europa, sorprende la enorme cantidad de autores cuyos nombres apenas han dejado unas líneas en obras tanto biográficas como prosopográficas de referencia. ${ }^{2}$ En este terreno, biobibliografías de calígrafos españoles como las conocidas de Cotarelo (1916), Rico (1903), San Vicente (1969) y Martínez Pereira (2006) se suman a las obras de consulta imprescindible para Inglaterra (Heal, 1931), Alemania (Doede, 1958), Francia (Mediavilla, 2005; 2006), Holanda (La Fontaine, 1976; Becker, 1997; Croiset, 1978) o Italia (Morison, 1962; Bonacini, 1953; Johnson, 1953; Marzoli, 1962, Erdmann et al., 2015).

Escrito según la indicación descriptiva técnica por un desconocido Ferdinand Geiler, es precisamente la autoría uno de los principales problemas de este brillante ejemplar. ${ }^{3}$ En realidad, esta muestra de Schönschriftbuch ("Libro de escritura bella" o "Libro de caligrafía") ilustra perfectamente la voluntad de presentar el virtuosismo caligráfico de su autor, a través de una excelente recopilación de un muestrario de letras y ejemplos desplegados primorosamente, de tal modo que permite reforzar la consideración de época dorada de la caligrafía otorgada a los siglos XVI y XVII. Sin data cronológica explícita, cabe situarlo en la segunda mitad del siglo XVI, habida cuenta de la mención hecha en el folio 46r al emperador Fernando I de Austria, entronizado en 1558 .

El códice, de neta inspiración germánica, presenta una enorme belleza en su factura formal y reproduce numerosos tipos y modelos de escritura, esencialmente libraria y sentada, algunas de considerable fantasía y abigarramiento geométrico y visual de gran elegancia, múltiples taraceadas y otras muchas usadas generalmente para códices de lujo. Los textos son en su práctica totalidad breves fragmentos de salmos o pasajes bíblicos y, a diferencia de otros ejemplos coetáneos similares, no se adentra en la práctica caligráfica

1 Göttingen (Baja Sajonia, Alemania); Niedersächsische Staats-und Universitätsbibliothek. (Quer $8^{\circ}$ ) Cod. Ms. Philos. 27 Cim: L’Art d'écrire. I Eine Probe der Kantzlungschrift von H. Ferdinand Geiler [sic], según el propio catálogo de la Universidad: Verzeichnis der Handschriften im Preussischen Staate. I. Hannover. 1: Göttingen 1 (Berlín: Verlag Von A. Bath, 1893), 142-143.

2 La exposición titulada Caligrafía española: el arte de escribir, mostrada desde septiembre de 2015 hasta comienzos de 2016 en la Biblioteca Nacional de España, es un interesante recordatorio de esta fiebre caligráfica que recientemente se ha instaurado. Por otra parte, proyectos de investigación como el que desarrolla Marc Smith (L'École nationale des chartes) en Francia, bajo el título Catalogue général des recueils de calligraphie gravés en France, 1560-1815, son exponentes de este necesario enfoque recopilatorio y exhaustivo.

3 En la obra de necesaria consulta, publicada por Werner Doede (1958), no aparece ninguna mención a este autor ni a ninguno con nombre similar. Tampoco en el magnífico trabajo sobre la escuela de calígrafos de Núremberg, con especial atención a los maestros Neudörffer y Fugger (Linke y Sauer, 2007). 
de minúsculas cursivas y documentales, tan propias de la voraz administración por escrito que define el burocrático Estado moderno.

La influencia centroeuropea es evidente, situando este ejemplar en la órbita de la caligrafía recopilada durante el pleno Renacimiento, con abundantes referencias gráficas a las escrituras góticas del cambio de siglo, en el que convivieron formas propias de la Baja Edad Media con otras heredadas de la tradición escrita romana, y también con otras coetáneas que definen ya el humanismo y su incuestionable claridad caligráfica y tipográfica. No cabe considerar este ejemplar, stricto sensu, un tratado de escritura o un manual para calígrafos, ya que, a diferencia de otros casos conocidos, éste es un códice que no presenta ningún atisbo formativo, didáctico o instructivo (Gasparri, 1979). Más bien nos hallamos ante una obra que se inscribe en una concepción formal de la escritura de élite, a través de la perfección caligráfica, elaborada no tanto para enseñar o instruir el desarrollo pautado de trazos como para mostrar ad electionem diferentes modelos escriturarios, aunque sin las habituales (y a veces controvertidas) designaciones de letras que suelen acompañar a este tipo de materiales. Podría tratarse de un muestrario para uso profesional interno de un pendolista, habida cuenta de su falta de indicadores publicitarios o de vocación explícita de dar a conocer esta obra.

Por otra parte, si fuera un encargo superior, como era habitual en las casas reales o en el seno de la nobleza, se hubiera consignado esta circunstancia con las loas habituales del oferente. Por el contrario, tanto el silencio y la escasez de referencias asociadas al quién, para quién y por qué de este manuscrito definen su singularidad. Un detalle no menor, como el hecho de que prácticamente todo el contenido de este códice se ejecutase sobre pergamino y sean frecuentes las muestras de chrysographia y argyrographia constituyen indicadores de su voluntad suntuaria, hermanando este ejemplar con otros de similares características y contexto.

\section{ОвJETIVOS y MÉtodo}

Este trabajo preliminar forma parte de un proyecto de investigación más amplio, en el que el autor de estas líneas prepara varias ediciones de manuscritos europeos inéditos, fechados durante los siglos XVI al XVII, los cuales constituyen ejemplos de virtuosismo caligráfico, con similares parámetros de elaboración y finalidad. Esta recuperación de testimonios caligráficos a 
través de ediciones críticas se suma a la inapreciable tarea legada por autores como Nicholas Barker (2009) o Stanley Morison (ed. 1990). Por su parte, John Ryder (1965) ya anotaba en 1965 una singular advertencia: "FACSIMILKE WANTING", indicación que actúa como verdadero estímulo para sacar adelante ediciones de obras que marcan hitos fundamentales en la historia de la caligrafía. De entre todas éstas destacarían las notables de Cesare Domenichi, Luca Orfei o Jacques de la Rue, así como los maravillosos cartones de Francesco Alunno di Ferrara, en la no menos lujosa edición a cargo del citado Barker (2009), sin perder de vista la célebre edición de los clásicos Mira Calligraphiae Monumenta del Getty Museum (Hendrix y Vignau, eds., 1992).

Como se ha señalado, el carácter inédito, hasta la fecha, de este códice Göttingen ms. Philos 27, obliga, en primer lugar, a realizar una descripción analítica aplicando un estudio pormenorizado, basándose en la propuesta metodológica realizada por el profesor Alberto Montaner Frutos (1999) en su Prontuario de bibliografía.

Junto a este objetivo formal-descriptivo que permite arrojar luz sobre aspectos de la llamada "fortuna del códice", un segundo objetivo es contextualizar este bello ejemplar en el seno de la alta cultura escrita, en la que se situaría junto a otros notables muestrarios caligráficos inéditos hasta nuestros días, elaborados en las cortes europeas durante los siglos XVI al XVII. ${ }^{4}$ Ejemplos todos ellos de imprescindible análisis para comprender los modos de adquisición de la competencia escrituraria virtuosa junto a manuales, tratados y otras vías que marcaron el panorama de la normalización en el aprendizaje caligráfico de los siglos modernos en Europa (Clayton, 2015). Ello nos autoriza a subrayar el interés del ejemplar concreto para su aplicación a dos ámbitos específicos: las competencias técnicas del profesional que gestiona fondo bibliográfico y documental antiguo, así como su estudio práctico, a modo de ejemplo relevante en el seno de la docencia paleográfica y caligráfica.

Es ahí donde se sitúa el tercer objetivo, ya que con este trabajo se busca proporcionar un ejemplo claro de la integración eficaz de tres facetas interrelacionadas y en continua retroalimentación: la docencia especializada en paleografía y caligrafía, la investigación en historia del libro y de la cultura escrita (faceta teórica), unida finalmente a la práctica caligráfica diaria para completar un perfil profesional integral e integrado (faceta práctica) (Navarro, 2014).

Por otra parte, no es ocioso resaltar que, precisamente, en aras a la conjunción de saberes (la tan buscada interdisciplinariedad), la comprensión de la materialidad del escrito es competencia imprescindible para quienes también se dedican a la ecdótica adentrándose en su textualidad (Montaner, 2012).

4 En la actualidad, estamos trabajando en otros manuscritos que constituyen excelentes muestras de escritura caligráfica del mismo periodo, con vistas a una próxima edición. 


\section{Descripción, análisis y comentario del códice}

Göttingen (Alemania), Niedersächsische Staats-und Universitätsbibliothek. (Quer $8^{\circ}$ ) Cod. Ms. philos. 27 Cim. Pergamino. ${ }^{5}$

[Autor] Ferdinand Geiler ${ }^{6}$

[Título facticio:] Schönschriften [=Bellas escrituras]

[Título formal:] f.1r: L'Art d'écrire. I Eine Probe der Kantzlungschrift von $\mathrm{H}$. Ferdinand[us] Geiler. ${ }^{7}$

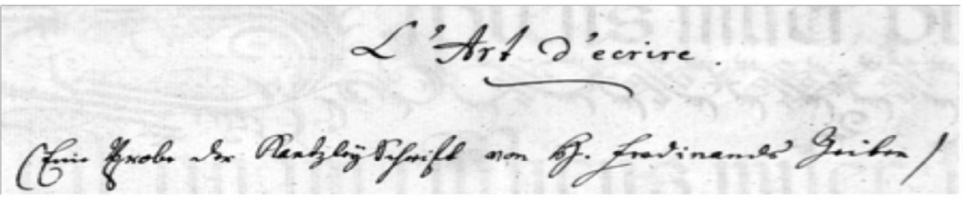

\section{Libro apaisado ( $320 \times 235 \mathrm{~mm})$}

Blanco. Hoja de respeto en papel.

f. 1r: L’Art d'écrire. I Eine Probe der Kantzlungschrift von H. Ferdinand Geiler [sic]. Papel, numeración original únicamente en las dos primeras hojas. El resto, con notables alternancias y lagunas, numeración contemporánea a lápiz en ángulo superior derecho.

El códice concluye en f. 62, en blanco y hasta esa marca, se incluyen 47 muestras de escritura diferente, todas de gran solemnidad y aparato.

5 Salvo indicación de las hojas de respeto en papel, todo el volumen está escrito sobre pergamino.

6 Según el propio catálogo de la Universidad: Verzeichnis der Handschriften im Preussischen Staate. I. Hannover. 1: Göttingen 1 (Berlín: Verlag von A. Bath, 1893), 142-143: En p. 142: [Anotación manuscrita del personal técnico de biblioteca]: Cim Philos. 27: "L’Art d'écrire. (Eine Probe der Kantzlungschrift von H. Ferdinand Geiler)”. Consultado el personal de la propia biblioteca para averiguar más datos sobre la proveniencia y particularidades de la incorporación de este ejemplar a la colección, no fue posible localizar detalles diferentes de los consignados en el propio catálogo de manuscritos y que reproduzco arriba. P. 143: " 8 " Philos 27. Pergament. 60 Bl. Zu 23,5: 32 cm. XVII. Jahrh. Schönschriften Jedes Blatt ist mit anderer Schrift beschrieben. Die Schriften sind mitunter sehr schön und kunstvoll. Meistens geben sie lateinische Bibelverse, doch Bl. 38 "Ferdinandus divina favente clementia electus Romanorum imperator" und Bl. 46 "Wir Ferdinand von G[ottes] [sic] gnaden erwelter römischer Kaiser".

7 Esta denominación de Kantzlungschrift/Cantzleyschbuch es la misma que encontramos en el ejemplar manuscrito de otro gran calígrafo centroeuropeo, Urban Wyss, y debe aclararse que los Kantzleybücher son más bien formularios notariales o secretarios de modelos de cartas, y no tanto manuales o tratados de bella escritura, caligrafía o Schönschrift. Urban Wyss (1553) (cfr. Johann Peter Zwengel, 1568). http://www.mdz-nbn-resolving.de/urn/resolver.pl?urn=urn:nbn:de:bvb:12-bsb10145119-2. 


\begin{tabular}{|c|c|c|c|c|}
\hline f. [3r] & $\begin{array}{l}\text { Humili I tas est u I } \\
\text { irtuso [sic] }\end{array}$ & $\begin{array}{l}\text { Letras capitulares } \\
\text { ornamentales } \\
\text { góticas tardías }\end{array}$ & $\begin{array}{l}\text { Elaboradas con } \\
\text { pluma de doble } \\
\text { trazo y tinta sepia. } \\
\text { Comienza con una } \\
\text { Hen sepia y oro }\end{array}$ & sion \\
\hline f. $[4 r]$ & $\begin{array}{l}\text { DOMINE } \\
\text { DOMINUS I } \\
\text { NOSTER QUAM I } \\
\text { ADMIRABILE }\end{array}$ & $\begin{array}{l}\text { Mayúsculas de ins- } \\
\text { piración lombarda. }\end{array}$ & $\begin{array}{l}\text { Negro. Interesante } \\
\text { pautado que } \\
\text { enmarca la caja } \\
\text { de escritura en } \\
\text { las tres líneas y, } \\
\text { especialmente, las } \\
\text { marcas de escri- } \\
\text { tura preparatoria } \\
\text { en lápiz }\end{array}$ & 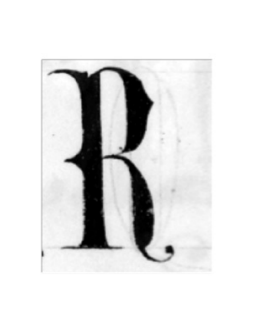 \\
\hline f. $6 r$ & $\begin{array}{l}\text { IN EXITV ISRA I } \\
\text { EL DE AEGYPTO: } \\
\text { I DOMVS IACOB. } \\
\text { I DE POPVLO BAR } \\
\text { I BARO }\end{array}$ & $\begin{array}{l}\text { Negro. Capitales } \\
\text { romanas }\end{array}$ & $\begin{array}{l}\text { Se aprecia el } \\
\text { mismo proceso } \\
\text { preparatorio a } \\
\text { lápiz que en el } \\
\text { ejemplo anterior } \\
\text { del f. } 4 \mathrm{r}\end{array}$ & 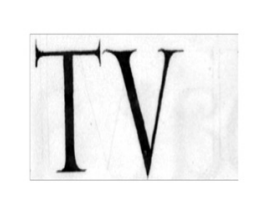 \\
\hline f. $[7 r]$ & $\begin{array}{l}\text { Lugebat autem lu } \\
\text { I dam israel planc } \\
\text { I tu magno: Et di I } \\
\text { cebat quomodo }\end{array}$ & $\begin{array}{l}\text { Negro. Letra redon- } \\
\text { da humanística } \\
\text { invertida para ser } \\
\text { leída con espejo }\end{array}$ & & 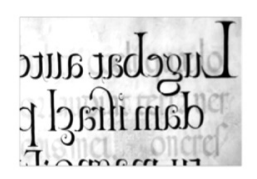 \\
\hline f. $[7 \mathrm{v}]$ & $\begin{array}{l}\text { En blanco, pero la } \\
\text { tinta se transpa- } \\
\text { renta ofreciendo la } \\
\text { lectura del anterior } \\
\text { texto en limpio }\end{array}$ & & & $\begin{array}{l}\text { dam ifracl planc } \\
\text { cu magho:Ecdi } \\
\text { ccharQuomodo }\end{array}$ \\
\hline f. $8 r$ & $\begin{array}{l}\text { Audite coeli quae } \\
\text { lolquor audiat terra } \\
\text { verlba oris mei } \\
\text { Concreslcat ut } \\
\text { pluuia doctrina }\end{array}$ & $\begin{array}{l}\text { Negro. Letra redon- } \\
\text { da, rotunda o "letra } \\
\text { de libros", según } \\
\text { la denominación } \\
\text { de Iciar }\end{array}$ & $\begin{array}{l}\text { Incorpora una A } \\
\text { y una C miniadas } \\
\text { en oro. Pautado a } \\
\text { punta seca }\end{array}$ & 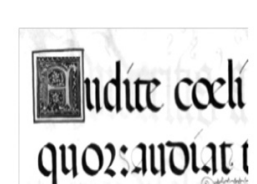 \\
\hline f. $19 r$ & $\begin{array}{l}\text { Ego in altissimus } \\
\text { habito et I thronus } \\
\text { meus in columna I } \\
\text { nubis superborum } \\
\text { et sublilmium colla } \\
\text { propria virtutlis }\end{array}$ & $\begin{array}{l}\text { Gótica taraceada } \\
\text { ejecutada con } \\
\text { pluma de doble } \\
\text { punta, ojos de letra } \\
\text { decorados con } \\
\text { alternancia de } \\
\text { blanco/negro. }\end{array}$ & $\begin{array}{l}\text { E capital inicial con } \\
\text { oro. Se perciben } \\
\text { vacilaciones. } \\
\text { Pautado a lápiz. }\end{array}$ & 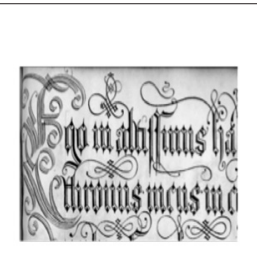 \\
\hline
\end{tabular}




\begin{tabular}{|c|c|c|c|c|}
\hline $\begin{array}{l}\mathrm{f} . \\
{[30 \mathrm{r}-} \\
31 \mathrm{v}]\end{array}$ & BONUM I STUDE & $\begin{array}{l}\text { Hoja doblada con } \\
\text { ejemplo de letra } \\
\text { mayúscula insular } \\
\text { con lacerías y de- } \\
\text { coración en azules } \\
\text { y rojos }\end{array}$ & & be. \\
\hline $\begin{array}{l}\text { f. } \\
{[36 r-} \\
37 v]\end{array}$ & $\begin{array}{l}\text { DEUS POTE I ST } \\
\text { OMNIA }\end{array}$ & $\begin{array}{l}\text { Hoja doblada } \\
\text { con dos líneas } \\
\text { de mayúsculas } \\
\text { ornamentales }\end{array}$ & $\begin{array}{l}\text { Inspiradas en } \\
\text { las capitulares } \\
\text { antropomorfas de } \\
\text { Vespasiano Am- } \\
\text { phiareo (1548). }\end{array}$ & 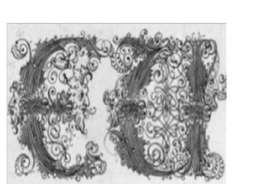 \\
\hline $\begin{array}{l}\text { f. } \\
{[44 r-} \\
45 v]\end{array}$ & $\begin{array}{l}\text { FORMA VII NO } \\
\text { PERIT }\end{array}$ & $\begin{array}{l}\text { Hoja doblada con } \\
\text { dos líneas de escri- } \\
\text { tura. Mayúsculas de } \\
\text { negro intenso. }\end{array}$ & $\begin{array}{l}\text { De nuevo, clara } \\
\text { influencia de Am- } \\
\text { phiareo }\end{array}$ & \\
\hline $\begin{array}{l}f . \\
{[46 r-} \\
47 v]\end{array}$ & $\begin{array}{l}\text { Wir Ferdinand von } \\
\text { Gottes gnalden } \\
\text { Erwelter Romis- } \\
\text { cher Kaiser }^{a}\end{array}$ & $\begin{array}{l}\text { Hoja doblada con } \\
\text { dos líneas de } \\
\text { escritura. Plata y } \\
\text { oro. Fraktur con } \\
\text { influencias de } \\
\text { Neudörffer y S. } \\
\text { Brechtel. Pautado a } \\
\text { lápiz. Muy similar a } \\
\text { las muestras de los } \\
\text { ff. 38v-39r }\end{array}$ & & \\
\hline
\end{tabular}

Cuadro 1. Selección de muestras de escritura incluidas en el Göttingen, Niedersächsische Staats-und Universitätsbibliothek. (Quer $8^{\circ}$ ) Cod. Ms. philos. 27 Cim: L'Art d'écrire

Todo el volumen en pergamino, salvo las hojas de respeto en papel verjurado y el f. 1 . Presenta marca de agua: escudo con corona real abierta, rematada de cinco flores de lis. En el campo del escudo, un cuerno de cazador colgante. En la base del escudo, las letras mayúsculas WR. La identificación de la marca de agua nos llevó a consultar a María Stieglecker, investigadora dentro del proyecto Wasserzeichen des Mittelalters (Institut für Mittelalterforschung der Österreichischen Akademie der Wissenschaften), a quien agradecemos sus desvelos. El resultado arrojó varios ejemplos muy aproximados a la marca de agua que reprodujimos a mano alzada. En concreto, existen cinco entradas, y especialmente una que guarda una semejanza casi total con la marca de agua de la hoja de guarda del manuscrito 
de Göttingen. Sus datos son interesantes desde el punto de vista cronológico (1663) y geográfico (molino papelero de Oberkirch, ciudad al sur de Alemania), elementos que acreditan que el uso de este papel como hoja de guarda fueron posteriores a la época en que el grueso del manuscrito en pergamino fue elaborado (ca. 1560).

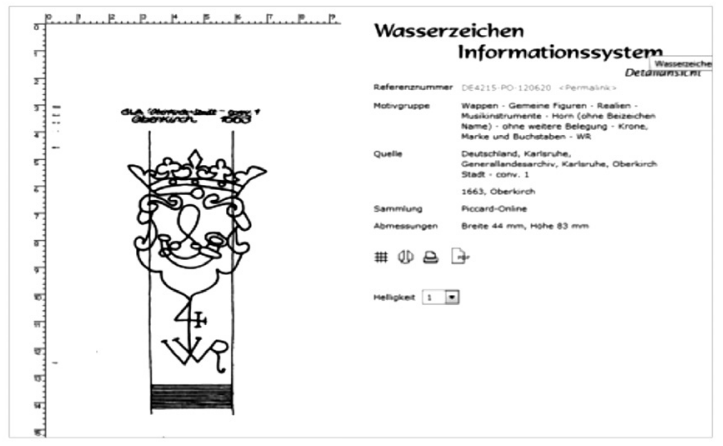

Fuente: Wasserzeichen Informationssystem (Deutsche Forschungsgemeinschaft), http://www.wasserzeichen-online.de/wzis/struktur.php?ref=DE4215-P0-120620

Buen estado de conservación, salvo ocasionales marcas de insectos bibliófagos (especialmente derméstidos a partir del f. 56 y ss.). El códice presenta una doble estructura: por un lado, uniones cosidos y, por otra, biniones pegados a tiras de pergamino, que es el que realmente se cose y adjunta a los demás, en aquellos casos en que el tamaño de la escritura requería un formato alargado superior al folio en pergamino corriente.

Encuadernación sobria en piel, posterior al manuscrito, sin ornamentación reseñable ni orlas. Siglo XVII. Lomo con 4 nervios. Florones dorados entre nervios, salvo en el segundo, donde se lee: LART I DE ICRIRE en capitales romanas empastadas. Restos de dorado en los cortes. Superlibros: tanto cubierta como contracubierta presentan un único escudo estampado en oro como marca de propiedad: escudo cuadrilongo de base levemente conopial, cortado. Primero, un león echado que sostiene en sus manos una flor de lis; segundo, tres medias flores de lis. Al timbre, corona abierta rematada por cinco florones visibles de hojas de acanto (tres grandes alternados con dos pequeños). El todo enmarcado por una láurea. El león echado, como figura heráldica infrecuente, nos pone cautelarmente sobre la pista de la familia Jacquet, asentada en Lyon durante la segunda mitad del siglo XVI en adelante (Mesnil, 1872). Gaspard Jacquet desarrolló una íntima conexión con el mundo escriturario y administrativo como secretario del rey de Francia y custodio de los sellos en el seno de la cancillería regia a comienzos del 
siglo XVII, circunstancia que también podría avalar la hipótesis de la propiedad de este ejemplar de tipos de escritura ornamental para su uso profesional (Tessereau, 1710: 318).

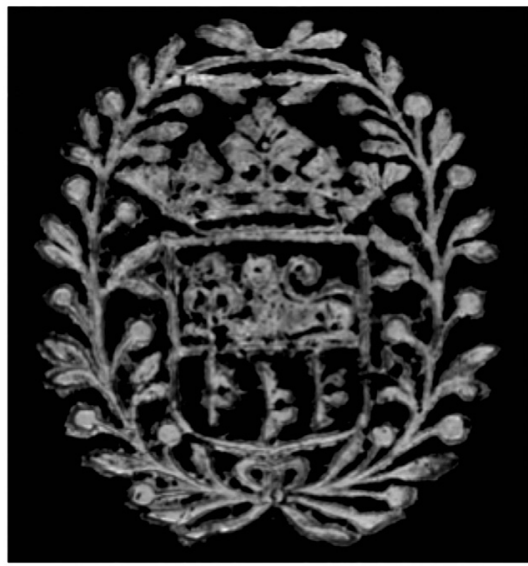

Superlibros: decoración heráldica de cubierta

Sin aditamentos de broches, bullones o cantoneras. Presenta dos tejuelos: uno en el lomo superior, pegado con la anotación: Cim. Otro en el lomo inferior, moderno, pegado. En azul: Cod. MS. Philos 27.

Ejemplar guillotinado en exceso, con resultados de pérdida de trazos en algunos folios. Por ejemplo, el 41r.

Sin sellos ni exlibris, salvo el fol. 2v, correspondiente a la biblioteca de la propia Universidad de Göttingen: EX I BIBLIOTHECA I REGIA ACAD.| GEORGIAE | AUG

Sin bibliografía ni estudios complementarios hasta la fecha.

\section{CONCLUSIÓN: MUESTRARIOS CALIGRÁFICOS COMO EXPONENTES} DE UNA CULTURA ESCRITA DE ÉLITE

La terminología encontrada hace agrupar estos singulares resultados caligráficos bajo las siguientes denominaciones, muchas veces empleadas como sinónimos en las descripciones bibliográficas (Croiset, 2016; Roth, 2010): Musterbuch, Schönschriften, Arts d'écriture, Schriftkunsbücher, Schreibkunst, Schönsreiben, Zierliche Schöne Schriften, Modus/Forma/Ars Scribendi, Spiegel 
der Schriftkunst, Schreib-Arth, etc. Aspecto de enorme importancia para establecer una futura clasificación de estos ejemplos caligráficos de los siglos XV al XVIII es el conjunto de características que definen su materialidad constitutiva (Castillo, 2002; Chartier y Hébrard, 2013: 12). El soporte empleado, los formatos (generalmente apaisados o a la italiana , para un mejor aprovechamiento y proyección estética del resultado caligráfico), la preparación de las cajas de escritura en complicados ejercicios de medición espacial, interlineado y equilibrio visual, combinación ajustada de líneas que obligan al ojo a seguir complejas secuencias rítmicas, integración de colores, etc., nos sumergen de lleno en las fases tanto preliminares como de ejecución final de estos ejemplares, claramente producidos para mostrar y demostrar.

Como el ejemplar aquí descrito, estas artes caligráficas son artefactos manuscritos muy apropiados para dar cauce a esa voluntad de exposición y regocijo, por los alardes de virtuosismo y pericia. Debe distinguirse así de otros resultados similares, en los que primó la voluntad formativa, explicativa o de alcanzar por vía de ejemplo ordenado y didáctica reposada la competencia en el arte de la pluma. Algunos superaron el estado manuscrito y fueron difundidos con éxito por vía de edición impresa. Esa preocupación normativa se acompañaría de un innegable deseo de trascender visualmente la mera comunicación del texto, en ocasiones hasta límites extremos que hacen peligrar la comprensión del mensaje y su legibilidad, en beneficio de una estética deslumbrante dentro de la dialéctica entre capacidad semántica y poder gráfico (Mediavilla, 2005). Esto se muestra señaladamente en algunas épocas y lugares, como la corte del emperador Maximiliano de Habsburgo. Una corte que alumbró como muestra representativa de estos códices caligráficos para uso particular el célebre Kalligraphiebuch de su esposa María, duquesa de Borgoña y de Brabante (König, ed., 2015). Materialmente eran ejemplares únicos, de notable ejecución y refinamiento, realizados bien por encargo, bien como obsequio galante, propios de un entorno de elevada cultura en el que, en ocasiones, los autores, calígrafos con acceso a las más altas instancias cortesanas, podían situarse cerca del poder. Éstos alternaban muchas veces su competencia administrativa como secretarios, pendolistas o maestros de escritura de la propia familia real o imperial, con otros encargos donde la escritura embellecida superaba la mera funcionalidad burocrática y se hermanaba con otras muestras artísticas suntuarias de pintores, escultores, joyeros, grabadores, etc., en un gusto integral por el arte refinado de los destinatarios, situados en la élite política o económica.

Junto a la corte de Maximiliano y María, cuyo impulso dado al esplendor de las artes y letras germánicas del primer renacimiento fue extraordinario, debería citarse el foco caligráfico irradiado desde la ciudad de Núremberg, 
con el magisterio ejercido por Johannes Neudörffer y sus importantísimos discípulos y seguidores, entre los que destacarían Christian Fabian Brechtel o Paul Franck (Linke y Sauer, 2007), o más tarde en las ciudades de Praga o Berna, con calígrafos de un barroquismo insuperable, como Johann Rudolf Sager o el afamado Jakob Hutzli (Rubi, 1988). Todo ello sin menospreciar los afamados tratados caligráficos holandeses posteriores, tanto de Jodocius Hondius y su Theatrum artis scribendi (1594), como de su coetáneo, el celebérrimo Jan van den Velde (1568-1623) y su extraordinario Spieghel der schrijfkonste (ca. 1609) (Croiset, 1984).

Por otra parte, conviene establecer otra diferencia. Así como los "secretarios o estilos de carta" fueron habituales ejemplos a los que acudir en toda Europa para reproducir el contenido de comunicaciones epistolares, siguiendo pautas comúnmente aceptadas (Egido, 1995; Serrano, 2014), estos "libros de bella escritura" ofrecen una funcionalidad práctica: la de servir como muestrarios de letra para reproducir en ambientes cortesanos, en entornos escolares o administrativos e incluso literarios-creativos (Wyss, 1553; Schramm, 1928). En los primeros prima la estructura y el contenido textual; en los segundos, la dimensión estética y la morfología de la escritura.

El manuscrito que aquí se estudia incorpora un explícito título en francés, Art d'écrire. Sin embargo, no es propiamente dicho un arte de escribir habitual. Como se ha señalado, no muestra ni una voluntad normativa, tampoco un conjunto de instrucciones, procedimientos o regulae para comprender el fundamento del ductus de cada tipo de letra ejecutada. Tampoco hay denominaciones o rótulos con terminología de tipos de letra, práctica más propia de los manuales o artes de escritura gestados con vocación didáctica o formativa que trataban de fijar las variaciones en las letras con su bautismo nominal más o menos afortunado (Frense, 1883-1887). En este sentido, cabría identificar semejanzas (pero sobre todo diferencias) con tempranos manuscritos que incorporaron múltiples tipos de escritura con sus denominaciones. Las célebres doce muestras de escritura fechadas entre 1400-1420 del maestro de escritura Johannes von Hagen sigue siendo uno de los más primitivos ejemplos caligráficos con denominación de las letras reproducidas, los cuales alcanzarán un desarrollo extraordinario durante los dos siglos siguientes (Roth, 2010; Schneider, 2005). ${ }^{8}$ Mayor alcance y repercusión tuvo el manuscrito Proba centum scripturarum diversarum, notable despliegue caligráfico del monje Leonhard Wagner (1454-1522) (Wehmer, 1963), ${ }^{9}$ en el que se ofrece una muestra de cada una de aquéllas, con su denominación específica, desde la rotunda hasta la

8 El original en Berlín, Staatsbibliothek-Preussischer kulturbesitz ms. Lat. Fol. 384, lo ha estudiado Norbert Schneider (2005). 
italicana formata, pasando por la bullicalis minor. También habría notables diferencias con otros ejemplos de artes de escritura o de letras anteriores que adornan la historia caligráfica del siglo XV, como el Ars scribendi del notario Gabriel Altadell, fechado hacia 1468, o el Ars literaria de Hartmann Schedel, estudiados ambos por Gimeno Blay (1993; 2015, en prensa). A ellas habría que sumar la Forma scribendi de Hugo de Spechtshart (1346) (Steinberg, 1940-1941), el Modus scribendi de la Abadía de Melk (s. XV) (Bischoff, 1939; Morison, 1940), el Tractatus in omnem modum scribendi (s. XV) (Gasparri, 1979), o el compendio de la Biblioteca de la Universidad de Praga (s. XV) (Palm, 1865). Hallazgos similares al que nos ocupa, como los alcanzados por Karin Schneider (1994) en Múnich también deben consignarse.

Otros muchos originales permanecen custodiados en colecciones tanto públicas como privadas. A los cuales se sumarían, como mínima selección final, la Colección de todas formas de letras assí antiguas como modernas... (1769), manuscrito de Antonio de Huerta, ${ }^{10}$ o los cuadernos caligráficos de los Morante, ${ }^{11}$ los Moya, ${ }^{12}$ los Zabala ${ }^{13}$ o el de Marcos Fernández de las Roelas ${ }^{14}$ incluidos en la mencionada exposición Caligrafía española: el arte de escribir (Biblioteca Nacional, España, septiembre de 2015-enero de 2016). Es también, sin ir más lejos y para concluir, el caso del notable muestrario caligráfico inédito de Tomás Solórzano conservado en la colección particular de Javier García del Olmo que sirve para cerrar estas páginas. ${ }^{15}$

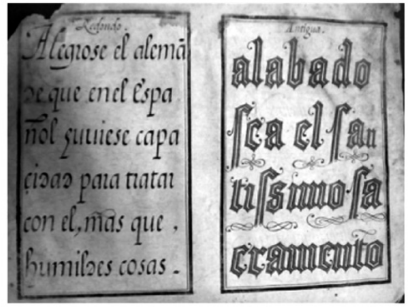

Thomas Solórzano escribe las formas de letra que en estas diez hojas se refieren en los años 1611 y 1613, Madrid, 1632-1633. Manuscrito en pergamino. Conservado en la colección García del Olmo/Vilas, Madrid.

10 BNE, mss. 9987.

11 Biblioteca de la Residencia de Estudiantes, Colección Rico y Sinobas, ms. R-549, 550, 551, 553.

12 Ibíd., ms. R-635.

13 Ibíd., ms. R-636.

14 BNE, mss. 22844.

15 Agradezco a mi amigo Javier García del Olmo su amabilidad y generosidad, al permitirme reproducir el fragmento de este brillante ejemplario caligráfico, que muy probablemente fue la versión previa a su entrega al tallador para la edición impresa que, hasta donde sabemos, nunca vio la luz. 


\section{REFERENCIAS}

Amphiareo, Vespasiano. 1548. Un novo modo d'insegnar a scrivere et formar lettere di più sorti che da altri non prima c' hora usate: novamente da frate Vespasiano minoritano trovato e da lui pur hora dato in luce. Florencia: Curtio Troiano di Navò.

Barker, Nicholas. 2009. The glory of the art of writing: The calligraphic works of Francesco Alunno of Ferrara. Los Ángeles: Costen Occasional Papers.

Becker, David. P. 1997. The practice of letters: The Hofer collection of writing manuals 1514-1800. Cambridge: Harvard College Library.

Bischoff, B., ed. 1939. Ein neuendeckter Modus Scribendi des xv Jabrbunderts aus der Abtei Melk. Berlín: Privatdruck der Setzmaschinen-Fabrik Monotype.

Bonacini, Claudio. 1953. Bibliografia delle arti scrittorie e della calligrafia. Florencia: Sansoni Antiquariato.

Castillo Gómez, Antonio. 2002. "El tiempo de la cultura escrita: a modo de introducción”, en Antonio Castillo, ed., Historia de la cultura escrita: del Próximo Oriente Antiguo a la sociedad informatizada. Gijón: Trea.

Chartier, Roger y Jean Hébrard. 2013. "Prólogo: morfología e historia de la cultura escrita”, en Armando Petrucci, ed., Alfabetismo, escritura y sociedad. Barcelona: Gedisa.

Clayton, Ewan. 2015. "La edad de oro del manual de escritura", en La historia de la escritura. Madrid: Siruela, pp. 134-136.

Cotarelo y Mori, Emilio. 1916. Diccionario biográfico y bibliográfico de calígrafos españoles. Madrid: Tipografía de la Revista de Archivos, Bibliotecas y Museos [Ed. facsímil Madrid: Visor, 2004].

Croiset van Uchelen, Anthony R. A. 2016. "Schreibmeisterbücher”, en Günther Pflug, ed., Lexikon des gesamten Buchwesens. Band I bis IX (komplett). Stuttgart: Anton Hiersemann, pp. 601-604.

Croiset van Uchelen, Anthony R. A. 1984. Deliciae: over de schrijfkunst van Jan van den Velde aan de hand van een inleiding op Van den Velde. Harlem: Enschede.

Croiset van Uchelen, Anthony R. A. 1978. Nederlandse Schrijfmeesters uit de eventiende eeuw: Tentoonstelling 16 Juni tot en Met 19 Augstus 1978. La Haya: Rijksmuseum Meermanno-Westreenianum.

Doede, Werner. 1958. Bibliographie deutscher Schreibmeisterbücher von Neudörffer bis 1800, Hamburgo: Ernest Hauswedell.

Egido, Aurora. 1995. "Los manuales de escribientes desde el Siglo de Oro. Apuntes para la teoría de la escritura", Bulletin Hispanique (Burdeos), no. 97: 67-94.

Erdmann, Axel, Alberto Govi y Fabrizio Govi. 2015. "Ars Epistolica: Communication in sixteenth century Western Europe: Epistolaries, letter writing manuals and model letter books, 1501-1600", European History Quarterly, no. 45: 549-551.

Frense, Charles de (Sieur du Cange) et al. (1883-1887), Glossarium medix et infimx latinitatis. Niort: L. Favre, VII, 370c.

Gasparri, Françoise. 1979. "L'enseignement de l'écriture à la fin du Moyen Âge: à propos du 'Tractatus in omnem modum scribendi', ms. 76 de l'abbaye de Kremsmüster", Scrittura e Civiltà, no. 3: 243-265.

Gimeno Blay, Francisco M. 2015 (en prensa). "Muestrario gráfico del otoño medieval: Ars literaria y Ars alphabetica de Hartmann Schedel”. Berlín: ponencia presentada en el XIX Coloquio Internacional de Paleografía Latina "Change in Medieval and Renaissance scripts and manuscripts", 16-18 de septiembre de 2015. 
Gimeno Blay, Francisco M. 1993. "Una aventura caligráfica: Gabriel Altadell y su De Arte Scribendi (ca. 1468)", Scrittura e Civiltà, no. 17: 207-270; 235, n0. 90.

Heal, Ambrose. 1931. The English writing masters and their copy-books: 1570-1800: a biographical dictionary and a bibliography. Cambridge: Cambridge University Pres.

Hendrix, Lee y Thea Vignau-Wilberg, eds. 1992. Mira calligraphiae monumenta: A Sixteenth-Century calligraphic manuscript inscribed by Georg Bocskay and illuminated by Joris Hoefnagel. Malibú: The Paul Getty Museum.

Hondius, Jodocus. 1594. Theatrum artis scribendi: varia summorum nostri seculi, artificiam [sic] exemplaria complectens, novem diversis linguis exarata, Amsterdam, ed. facs. Nieuwkoop: Miland, 1969.

Johnson, A.F. 1950. Bibliography of italian Writing-Books of the XVI Century [s.d.].

König, Eberhard, ed. 2015. Das Kalligraphiebuch der Maria von Burgund. Ein Kalligraphiebuch mit feinsten Federzeichnungen.: Handschrift II 845 der Bibliothèque royale de Belgique in Brüssel. Lucerna: Quaternio.

La Fontaine Verwey, Herman de. 1976. “The golden age of dutch calligraphy”, en J.P. Gumbert y M. J. M. De Haan, eds., Miniatures, script, collections. Essays presented to G.I Lieftinck. Ámsterdam: Brill, pp. 69-78.

Linke, Oliver y Christine Sauer. 2007. Zierlich Schreiben: Der Schreibmeister Johann Neudörffer d.̈̈., und seine Nachfolger in Núrnberg. Núremberg: Stadtbibliothek.

Martínez Pereira, Ana. 2006. Manuales de escritura de los Siglos de Oro: repertorio crítico y analítico de obras manuscritas e impresas. Mérida: Editora Regional de Extremadura.

Martínez Pereira, Ana. 2004. "El arte de escribir de Alonso Martín del Canto (1544)”, en Pedro Cátedra y María Luisa López Vidriero, dirs., La memoria de los libros. Estudios sobre la historia del escrito y de la lectura en Europa y América. Salamanca: Instituto de Historia del Libro y de la Lectura, pp. 201-214.

Martínez Pereira, Ana. 2003. "Un calígrafo español en la corte de D. Joao V: Marcos de las Roelas y Paz", Península. Revista de Estudios Ibéricos, 0: 355-368.

Martínez Pereira, Ana y Emilio Torné. 2007. “Arte subtilissima, 1550: teoría y práctica de la escritura en la edición más perfecta de la obra de Juan de Iciar”, en Juan de Iciar, Arte sutilísima, por la cual se enseña a escribir perfectamente, ed. Fermín de los Reyes. Madrid: Editorial Complutense, 35-64.

Marzoli, Carla C. 1962. Calligraphy, 1535-1885: A collection of seventy-two writing-books and specimens from the Italian, French, Low Countries and Spanish schools, catalogued and described. Milán: La Bibliofilia.

Mediavilla, Claude. 2006. Histoire de la Calligraphie Française. [S.1.]: Albin Michel.

Mediavilla, Claude. 2005. Caligrafía: del signo caligráfico a la pintura abstracta. Valencia: Campgráfic.

Mesnil, Edmond Révérend du. 1872. Armorial Historique [...]. Lyon: Aimé Vingtrinier (cfr. Jacques Pachot, <http://www.armorialdelain.fr/jacquet-2.html>).

Montaner Frutos, Alberto. 2012. "El criterio frente al dogma: cuestiones epistemológicas al hilo de los estudios medievales y renacentistas", en Natalia Fernández Rodríguez y María Fernández Ferreiro, coords., Literatura medieval y renacentista en España: líneas y pautas. Salamanca: SEMyR, pp. 143-175.

Montaner Frutos, Alberto. 1999. Prontuario de bibliografía. Gijón: TREA.

Morison, Stanley. 1962. Calligraphy: 1535-1885. A selection of seventy-two writing books and specimens from the Italian, French, Low Countries and Spanish schools, catalogued and described. Milán: La Bibliofilia. 
Morison, Stanley, ed. 1990. Early Italian Writing-Books: Renaissance to Baroque, ed. Nicholas Barker. Verona: Valdonega.

Morison Stanley y B. Bischoff, eds. 1940. A fifteenth century "Modus Scribendi" from the Abbey of Melk. Cambridge: Cambridge University Press.

Navarro Bonilla, Diego. 2014. "Caligrafía: arte, comunicación y modernidad”, Archivamos: Boletín ACAL, 93 (3): 29-36.

Palm, H. (1865), "Eine Anweisung zur Kalligraphie aus dem 15 Jahrhundert", Anzeiger für Kunde der deutschen Vorzeit, 12 (2): 49-53; 13 (3): 89-92.

Rico y Sinobas, Manuel. 1903. Diccionario de calígrafos españoles. Madrid: Imp. Sucesores de Jaime Ratés.

Roth, Michael. 2010. "Johan Neudörffer der Ältere und der Beruf des Schreibmeisters”, en Schrift als Bild. Berlín: Kupferstichkabinett; Staatliche Museen zu Berlin, pp. 110-122.

Rubi, Christian, ed. 1988. Alte Berner Scbreibkunst: Jakob Hutzli: Das Gülden ABC. Berna: Benteli.

Ryder, John. 1965. Lines of the alphabet in the sixteenth century. Londres: Stellar Press-The Bodley Head.

San Vicente Pino, Ángel. 1969. "Sobre algunos calígrafos del Bajo Renacimiento en Zaragoza”, en Suma de estudios en homenaje al Dr. Canellas López. Zaragoza: Universidad de Zaragoza, pp. 909-951.

Schneider, Karin. 1994. "Ein Schreiblehrbuch des frühen 16. Jahrhunderts”, BFB, 22: 177-183.

Schneider, Norbert. 2005. "Die Schriftproben des Schreibmeisters Johannes von Hagen...", Mittellateinisches Jabrbuch, 40 (3): 445-454.

Schramm, Albert. 1928. "Das Schreibbuch des Urban Wyss”, Archiv für Schreib-und Buchwesen 2: 40-44.

Serrano Sánchez, Carmen. 2014. “'Secretarios de papel’: Los manuales epistolares en la España moderna (siglos XVI-XVII)”, en Antonio Castillo Gómez y Verónica Sierra Blas, dirs., Cinco siglos de cartas. Historia y prácticas epistolares en las épocas moderna y contemporánea. Huelva: Universidad de Huelva, pp. 77-96.

Steinberg, H. (1940-41), “The Forma Scribendi of Hugo Spechtshart”, Transactions of the Bibliographical Society 21 (1): 264-278.

Tessereau, Abraham (1710), Histoire chronologique de la grande Chancelerie de France. París: Pierre Emery.

Wehmer, Carl. 1963. Leonhard Wagners Proba centum scripturarum. Faksimile und Kommentar. Leipzig: [s.ed.].

Wyss, Urban. 1553. Ein Schön Cantzleysch Tittelbuch inn Reden Vnd Schreybenn nach rettorischer Ardt. [s.n.] [ed. facs., Leipzig: Fritz Juntke, 1979].

Wyss, Urban. 1549. Das Schreibbuch des Urban Wyss. Libellus valde doctus et elegans sententiarum genera complectens. Zúrich H. Kienzle [ed. facs. Basilea: A. Schramm, 1928].

Zwengel, Johann Peter. 1568. New gross formular und vollkommen Cantzley Buch [...], Fráncfort: [s.ed.]. 
Agradezco a todo el personal de la Biblioteca de la Universidad de Göttingen, así como a Martina Clemen su inestimable ayuda, amabilidad y atención profesional en la gestación de estas páginas, así como al profesor Alberto Montaner (Universidad de Zaragoza), por sus siempre brillantes observaciones.

Para citar este texto:

Navarro, Diego. 2018. "Manuscritos caligráficos en fondos antiguos: análisis del ms. Göttingen Schönschriften (ca. 1560)". Investigación Bibliotecológica: archivonomía, bibliotecología e información 32 (76): 149-165. http://dx.doi.org/10.22201/iibi.24488321xe.2018.76.58002 